\title{
Gene expression alterations induced by low molecular weight heparin during bowel anastomosis healing in rats
}

\author{
Natalia Krześniak ${ }^{1}$, Agnieszka Paziewska², Tymon Rubel ${ }^{3}$, Magdalena Skrzypczak², \\ Michał Mikula ${ }^{4}$ Artur Dzwonek ${ }^{4}$, Krzysztof Goryca ${ }^{2}$, Lucjan S. Wyrwicz ${ }^{3}$, Dorota Jarosz ${ }^{2}$, \\ Daniel Laubitz ${ }^{5}$ Marek Woszczyński ${ }^{6}$, Krzysztof Bielecki ${ }^{1}$ and Jerzy Ostrowski ${ }^{2,4, \bowtie}$
}

${ }^{1}$ Department of General and Gastrointestinal Tract Surgery, Medical Center for Postgraduate Education, Warszawa, Poland; ${ }^{2}$ Department of Gastroenterology and Hepatology, Medical Center for Postgraduate Education, Warszawa, Poland; ${ }^{3}$ Laboratory of Bioinformatics and Systems Biology, Maria Sklodowska-Curie Memorial Cancer Center and Institute of Oncology, Warszawa, Poland; ${ }^{4}$ Department of Gastroenterology, Maria Sklodowska-Curie Memorial Cancer Center and Institute of Oncology, Warszawa, Poland; ${ }^{5}$ The Kielanowski Institute of Animal Physiology and Nutrition, Polish Academy of Sciences, Warszawa, Poland; ${ }^{6}$ Department of Animal Genetics, Maria Sklodowska-Curie Memorial Cancer Center and Institute of Oncology, Warszawa, Poland

Colon anastomosis is therapeutically challenging because multiple, usually undetectable factors influence a spectrum of repair mechanisms. We hypothesized that low molecular weight heparins, routinely administered perioperatively, may differentially affect gene expression related to colon healing. Twenty pairs of untreated and enoxaparin-treated rats underwent left-side hemicolectomy with a primary end-to-end anastomosis. Normal colon and anastomotic bowel segments were resected on day 0 and on days $1,3,5$, and 7 after surgery, respectively. Serial anastomosis transverse cross-sections were evaluated microscopically and by microarray (Rat Genome 230 2.0, Affymetrix). Differentially expressed probe sets were annotated with Gene Ontology. We also examined the influence of enoxaparin on fibroblast proliferation and viability in vitro. Among the 5476 probe sets, we identified differential expression at each healing time point, yielding 79 subcategories. Most indicated genes were involved in wound healing, including multicellular organismal development, locomotory behavior, immune response, cell adhesion, inflammatory response, cell-cell signaling, blood vessel development, and tissue remodeling. Although we found no intensity differences in histological features of healing between enoxaparin-treated and control rats, treatment did induce significant expression changes during early healing. Of these changes, 83 probe sets exhibited at least twofold changes and represented different functional annotations, including inflammatory response, regulation of transcription, regulation of apoptosis, and angiogenesis. Fibroblast culture confirmed an anti-viability effect of enoxaparin. Enoxaparin affects colon wound-related gene expression profiles, but further studies will resolve whether heparin treatment is a risk factor after intestinal surgery, at least in some patients.

Keywords: wound healing, heparins, gene expression, microarrays

Received: 16 August, 2010; 28 December, 2010; accepted: 21 January, 2011; available on-line: 18 March, 2011

\section{INTRODUCTION}

Wound repair activates multiple biological pathways that can restore tissue integrity in a precise sequence of events and consists of three overlapping phases: inflam- mation, formation of new tissues, and remodeling. As a consequence, wound repair is an effect of synchronized hemostasis, acute and chronic inflammation, and proliferation, differentiation, and migration of various cell types augmented with the synthesis of extracellular matrix proteins and collagen fiber arrangement. Collagen is the most essential basal, skeletal protein employed in healing processes. It is synthesized by proliferating fibroblasts to fill wounds and finally form a stable scar. Production of collagen and remodeling are initiated in the days after the wound occurs, but inflammation and formation of granulated tissue occur immediately and continue for several weeks. Although the above-mentioned processes occur in a highly orchestrated multiplestep procedure, specific actions overlap in the period of the first few days to weeks after wounding. Finally, most wounds are repaired in a fibrotic healing process that produces a scar (Gurtner et al., 2008).

Anastomosis leakage is one of the most serious complications of abdominal surgery. Although repair of an anastomotic wound results in a relatively common final outcome, a number of local, systemic, and external factors can influence the healing process. This multifactorial influence constitutes a considerable therapeutic challenge for surgery. Even if anastomosis is performed under optimal conditions, the risk of dehiscence remains as high as 3-20\% (Lustosa et al., 2002; Makela et al., 2003; Schmidt et al., 2003; Stumpf et al., 2005; Eberl et al., 2008). Such complications can lead to prolonged hospitalization and are associated with increased morbidity (Makela et al., 2003).

Thrombophylaxis using low molecular weight heparins (LMWHs) is routinely administered to all patients during the perioperative period. Nevertheless, unfractionated heparin (UFH) and LMWH can have adverse effects on the healing process. The antiproliferative effects of UFH and LMWH have been documented in fibroblasts (Tiozzo et al., 1991; Carroll et al., 2003), endothelial cells (Khorana et al., 2003), osteoblasts (Street et al., 2000) and vascular smooth muscle cells (Lake et al., 2003). Enoxa-

e-mail: jostrow@warman.com.pl

Abbreviations: LMWH, low molecular weight heparins; UFH, unfractionated heparin; VEGF, vascular endothelial growth factor; FBS, fetal bovine serum; $\mathrm{H} / \mathrm{E}$, hematoxylin and eosin; FDR, false discovery rate; $P C A$, principal component analysis; PMNs, polymorphonuclear cells; RMA, Robust Multi-array Average 
parin, an LMWH, influences viability, proliferation, and apoptosis of osteoblasts in culture and also delays fracture repair in animals (Street et al., 2000a; 2000b). Other studies have indicated that only UFH but not LMWH causes such bone defects in rabbits (Kock et al., 2002).

Both UFH and LMWHs alter the bioavailability and activity of growth factors. Platelet-derived growth factor, basic fibroblast growth factor, and vascular endothelial growth factor (VEGF) are all involved in wound repair and are bound and inactivated by heparins (Lane et al., 1989). Angiogenesis directly associated with VEGF activity is necessary for formation of granulated tissue during proper wound healing. LMWHs affect fibrin structure and inhibit angiogenesis in vitro (Collen et al., 2000). Heparin blocks fibroblast collagen production in culture, which delays wound maturation and consequently postpones final healing (Ferrao et al., 1993). Although a number of studies have sought to identify a broad spectrum of the biological effects of heparin, many challenging questions remain unanswered. Here we address the problem of whether or not heparins can modify the healing process of bowel anastomoses.

The study aim was to determine if and how administration of LMWHs in the perioperative period affects bowel anastomosis healing in a rat model (KrzesniakWszola, 2007). In this study, we present the results of global gene expressional profiling to elucidate a spectrum of molecular alterations. In addition, we examined the influence of enoxaparin on the proliferation and viability of fibroblasts in vitro.

\section{MATERIALS AND METHODS}

Cells. Human dermal scarified fibroblasts of the continuous lines (fetal and adult, about 20th passage) were cultured. Harvested cells were divided into portions and frozen. For each experiment, a new frozen cell portion was cultured in plastic cell culture flasks in Eagle's modified medium supplemented with 10\% fetal bovine serum (FBS), $2 \mathrm{mM}$ glutamine, penicillin $(100 \mathrm{U} / \mathrm{ml})$, and streptomycin $(0.01 \%)$ at $37^{\circ} \mathrm{C}$ and humidified with a $6 / 94 \% \mathrm{CO}_{2} /$ air mixture. Cells were routinely subcultured using trypsin solution. Enoxaparin was obtained as a pharmaceutical product and dissolved in phosphatebuffered saline.

Cell proliferation assay. Cell growth was determined by incorporation of $\left[{ }^{3} \mathrm{H}\right]$ thymidine into the DNA of proliferating cells. Exponentially growing cells were harvested, seeded in 96-well plates $\left(1.4 \times 10^{5}\right.$ and $2 \times 10^{5}$ of adult and fetal fibroblasts per well, respectively), and grown in a medium containing 10\% FBS. After $24 \mathrm{~h}$, cells were supplemented with fresh medium without or with enoxaparin in one of five concentrations $(20,2,0.2,0.02$, or $0.002 \mathrm{U} / \mathrm{ml})$. After $48 \mathrm{~h}, 0.1 \mu \mathrm{Ci}$ of $\left[{ }^{3} \mathrm{H}\right]$ thymidine was added to each well, and cells were cultured for an additional $24 \mathrm{~h}$. Media were changed daily.

Another set of experiments was performed on fibroblasts subjected to $24-\mathrm{h}$ serum deprivation (1\% FBS) and subsequently supplemented with $10 \%$ FBS and enoxaparin for 3 days. Three independent experiments were performed for each cell line, and all assays were repeated in octuplicate. Results were expressed as the percentage of control cells (means \pm S.D.) and analyzed using analysis of variance (ANOVA), MannWhitney U, or Kruskal-Wallis tests, depending on the data distribution. Means were considered to be different if $P<0.05$.
Cell apoptosis/necrosis assay. Exponentially growing cells were seeded in eight-chamber culture slides in medium containing 10\% FBS and grown until 50\% confluence was obtained. After $24 \mathrm{~h}$ of serum deprivation (1\% FBS), cells were supplemented with fresh medium containing enoxaparin in a range of concentrations as described above for 2 days. Media were changed daily. Finally, the adherent cells were stained on chamber slides with fluorescein-conjugated annexin $\mathrm{V}$ and propidium iodide using Annexin-V-FLUOS Staining Kit (Roche) according to the manufacturer's instructions. Fluorescence of individual cells was measured using fluorescence microscopy. The percentage of annexin V-and propidium iodide-stained cells within a minimum of 800 cells was determined. All experiments were performed in three independent repetitions. Results were expressed as a percentage of control cells (means \pm S.D.) and were statistically analyzed using ANOVA and Kruskal-Wallis tests. Means were considered to be different if $P<0.05$.

Animals. Forty male Wistar rats (weight 270-300 g, age approx. 3 months) were used in the study. The rats were housed under standard conditions and permitted free access to water and to standard food pellets. All procedures were reviewed and approved by the local ethics committee.

Operative procedure. Rats were assigned to two groups. In one group of 20 animals, enoxaparin was injected subcutaneously at a concentration of $0.5 \mathrm{mg} / \mathrm{kg}$ $24 \mathrm{~h}$ before the operation and each day after the operation. The other 20 animals were injected on the same time course with saline and served as controls. Anesthesia was induced by intramuscular ketamine hydrochloride, $50 \mathrm{mg} / \mathrm{kg}$ and xylazine, $5 \mathrm{mg} / \mathrm{kg}$. For each surgery, a median laparotomy of approx. $5 \mathrm{~cm}$ was made, and rats underwent a left-side hemicolectomy, with a primary end-to-end anastomosis of the left colon, approx. $3 \mathrm{~cm}$ proximal to the peritoneal reflection. The anastomosis was made with one-layer interrupted sutures using Maxon 7.0; fascia and skin were closed separately with a continuous Dexon 3.0 suture. At $0,1,3$, 5, or 7 days after surgery, the 8 rats were sacrificed and the $4-\mathrm{cm}$ anastomotic bowel segments were resected, the adherent tissues removed, and the bowel lumen cleaned with saline. Then, the excised segments were snap frozen and stored in liquid nitrogen. Before preparation, the longitudinal and transverse diameter of all resected colon specimens with an anastomosis were measured.

Microscopic analysis. Serial cryostat transverse cross-sections were collected from normal bowel and anastomoses. The four upper and four lower cryosections were stained with routine hematoxylin and eosin $(\mathrm{H} / \mathrm{E})$, and the remaining tissues were used as a source of RNA for the molecular analyses. Microscope slides were evaluated twice by the same experienced pathologist (D.J.) in a blind fashion, and parameters of the anastomotic healing (necrosis, granulation tissue, acute and chronic inflammatory cells) were scored in four stages of a semi-quantitative scale adapted from Kuzu et al. (1998): $0=$ negative, $1=$ slight, $2=$ moderate, and $3=$ massive . Edema was evaluated as the ratio of wall thickness at the anastomosis to the mean thickness of the normal colon from ends of resected segments, as follows: $0=$ negative; $1=$ the wall thickness ratio below $1.5 ; 2=$ below 2.0 ; and $3=$ over 2.0.

Sample preparation and microarray hybridization. Total RNA was extracted from cryostat-cut transverse cross-sections using the RNeasy Mini Kit (Qiagen) according to the manufacturer's protocol. Gene expression 
analyses were carried out using Affymetrix oligonucleotide microarrays (GeneChip Rat Genome 230 2.0) as described previously (Ostrowski et al., 2007).

To obtain gene expression measurements, the extraction of probe-level data was performed with a standard GC-RMA algorithm (Wu et al., 2004) for background correction and summarization steps and LVS algorithm (Gentleman et al., 2004) for normalization based on a least-variant set of probe sets. The calculations were performed with BioConductor (Affymetrix, 2002) packages grma (version 2.10.0) and FLUSH.LVS.bundle (version 1.1.1). The quality-control procedure was based on MAS5.0 extracted information, using thresholds suggested by Affymetrix (Affymetrix, 2002); as a consequence, one of the arrays was rejected because of poor quality. The internal consistency of data sets was also tested using principal component analysis (PCA), normalized unscaled standard error plots, and relative log expression plots.

For data filtration, we selected the probe sets exhibiting signal intensity above the threshold in at least $10 \%$ of samples. The threshold was established by fitting Gaussian functions to the two peaks observed in the histogram of expression values generated by the GC-RMA algorithm with LVS normalization.

Unsupervised average-linkage hierarchical clustering with the Pearson correlation coefficient-based distance metric and PCA were used for a graphic summary and evaluation of relationships between studied groups.

To establish gene expression profiles of the healing process, differentially expressed probe sets in the pairwise comparisons between the day 0 group and groups from the subsequent days after surgery (days 1, 3, 5, and 7) were identified using $t$-tests. ANOVA was also used for a multi-class comparison. The resulting $P$ values were adjusted for multiple testing using the BenjaminiHochberg procedure that controls a false discovery rate (FDR) (Benjamini \& Hochberg, 1995). The FDR threshold was set to 0.001, and only probe sets exhibiting a minimum two-fold change in mean relative expression were included in the gene lists. Cluster analysis of probe sets exhibiting differential expression at least at one time point was also performed. The probe sets were divided into groups of distinct expression patterns by an evolutionary-driven k-means clustering algorithm with a distance metric derived from the Pearson correlation coefficient. The selection of the number of clusters was based on visual inspection of the dendrogram resulting from an average-linkage hierarchical clustering procedure executed prior to the main cluster analysis.

In the heparin-treated vs control groups comparison, differential probe sets at each time point were identified with t-tests. Because of the expected small differences between expression profiles, permissive criteria were used for the feature selection step: the threshold for the $P$ values was set equal to 0.01 , which was accompanied by the requirement of at least a 1.25-fold change in expression. No FDR control procedure was used. A two-way ANOVA was also performed with the effect of heparin treatment as the first factor and day of specimen collection as the second.

Both statistical and cluster analyses were performed using a proprietary software working in the MATLAB (MathWorks) environment.

Functional analyses of gene expression by Gene Ontology. Differentially expressed probe sets were annotated with Gene Ontology (GO) terms (version 2.0.2) (Ashburner et al., 2000) using the Bioconductor packages rat2302 (version 2.0.1, Affymetrix Rat Genome 2302.0 Array Annotation Data) and package annotate (version 1.16.1). The significance of differential representation of GO terms between specified lists of probe sets was determined by the hypergeometric test implemented in GOstats (version 2.4.0) (Falcon et al., 2007). $P$ values returned by GOstats were corrected for testing of multiple hypotheses with the Benjamini-Hochberg method implemented in an $\mathrm{R}$ environment (version 2.6.1, The R Foundation for Statistical Computing; http://www.rproject.org). Adjusted $P$ values of less than 0.01 were considered significant.

Supplementary data. All gene expression data in MIAME complementary formats, supplementary figures and tables are available at http://integromics.pl/files/ Enoxaparine/

\section{RESULTS}

\section{Enaxoparin inhibits viability of cultured fibroblasts}

Studies performed on fetal and adult dermal scarified fibroblasts treated with an increasing concentration of enoxaparin showed that the highest concentrations of enoxaparin inhibited incorporation of ${ }^{3} \mathrm{H}$-thymidine into DNA in serum-deprived cells (in adult and fetal fibroblasts, a $30 \%$ and $15 \%$ decrease for $2 \mathrm{U} / \mathrm{ml}$ enoxaparin and $43 \%$ and $15 \%$ for $20 \mathrm{U} / \mathrm{ml}$ enoxaparin, respectively) (Fig. 1A). When exponentially growing cells were used, the same effect of the highest enoxaparin concentrations was observed only in adult fibroblasts $19 \%$ and $35 \%$ decrease for 2 and $20 \mathrm{U} / \mathrm{ml}$ enoxaparin, respectively) (Fig. 1B). Notably, at the lowest concentration $(0.002 \mathrm{U} / \mathrm{ml})$, enoxaparin enhanced proliferation of fetal fibroblasts (Fig. 1).

In repeated and quantified experiments, treatment with enoxaparin for $48 \mathrm{~h}$ did not change the level of early apoptosis but caused a statistically significant increase of late apoptosis and/or necrosis of both fibroblast lines, even with the lowest concentrations used (Fig. 2).

\section{Microscopic analysis of anastomotic healing}

Histological evaluation was performed to control the relative content of anastomosis repair-related changes with a series of cryostat transverse cross-sections. Upper cryosections represented colon sections taken approx. $4 \mathrm{~mm}$ from the anastomosis, while lower sections were cut out from a closer distance to the anastomosis. The sections were stained with $\mathrm{H} / \mathrm{E}$, and microscopic parameters of the healing were evaluated in a blind fashion on a semi-quantitative scale. Because histological alterations evaluated at both anastomosis sites of each excised segment showed no obvious differences (not shown), their scores were considered together during statistical analysis.

The histological examination of the anastomoses showed different features of anastomotic repair processes, which varied depending on the day after the operation (Table 1). From day 1, specimens exhibited massive edema and swelling of the colonic anastomosis, discontinuity of the mucosal layer, and low to moderate necrosis of the mucosal and submucosal layers with areas of infiltration by polymorphonuclear cells (PMNs) and lymphocytes.

In the samples obtained on the third day of healing, all the described microscopic parameters had increased in intensity. The bowel walls at the anastomotic site showed massive, full-thickness mucosal and submucosal 
Table 1. The pair-wise semi-quantitative comparisons of microscopic healing parameters estimated in two series of transverse crosssections of anastomosis sites that were obtained from four enoxaparin-treated and four control animals on days 1 , 3, 5, and 7 after surgery

Data represent means \pm standard deviation.

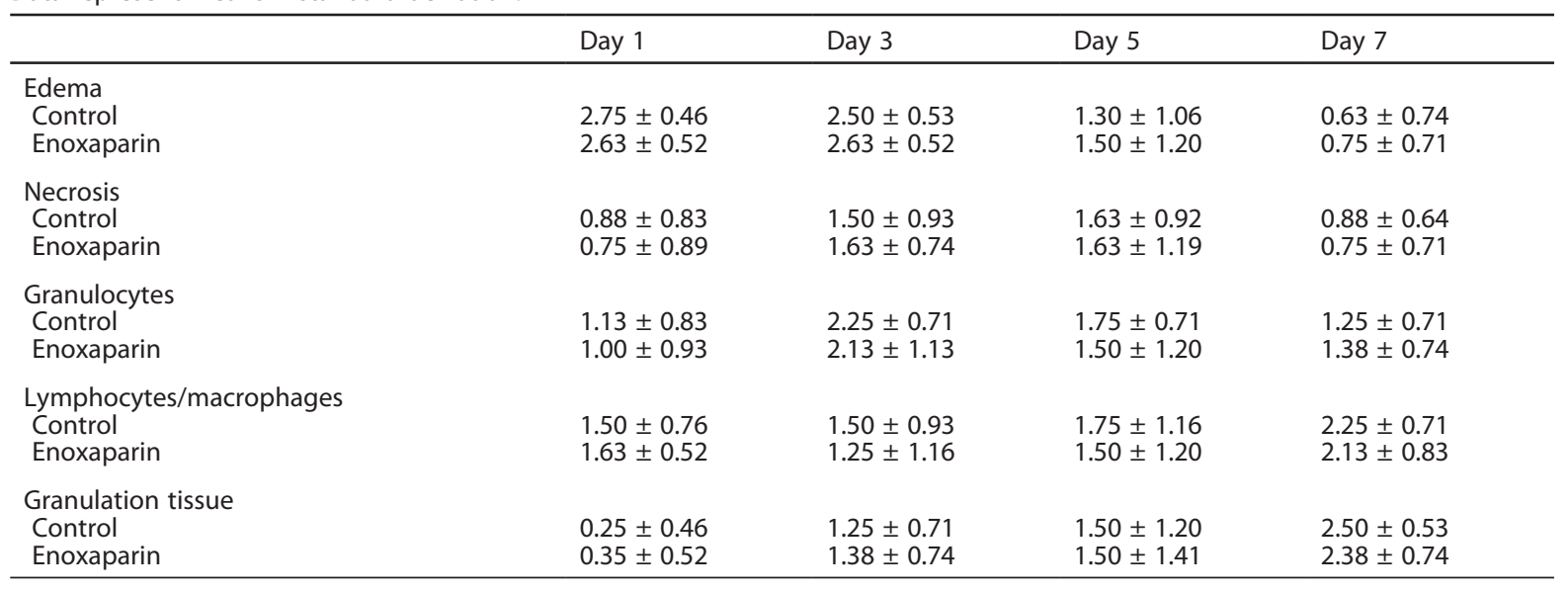

A.

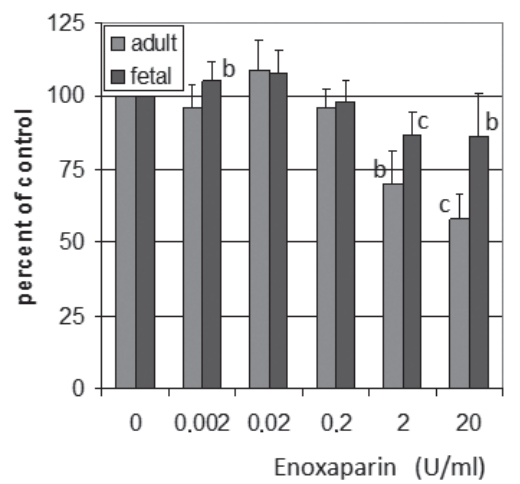

B.

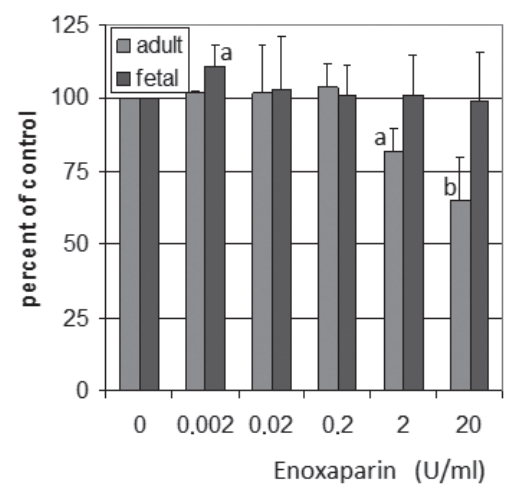

Figure 1. The effects of enoxaparin on proliferation of human fetal and adult dermal scarified fibroblasts, determined by ${ }^{3} \mathrm{H}$-thymidine incorporation

Panel A. Cells were grown for $24 \mathrm{~h}$ in 96-well plates in Eagle's modified medium containing $10 \%$ FBS and then were exposed to varying concentrations $(20,2,0.2,0.02$, and $0.002 \mathrm{U} / \mathrm{ml}$ ) of enoxaparin for 3 days. Panel B. 24-hour serum-starved fibroblasts were supplemented for 3 days with $10 \%$ FBS and with or without enoxaparin. $0.1 \mu \mathrm{Ci}$ of ${ }^{3} \mathrm{H}$-thymidine was added to each well for $24 \mathrm{~h}$ before cellular DNA was collected and radioactivity determined by scintillation counting. Three independent experiments were performed for each cell line. Results were expressed as the percentage of control cells (means \pm S.D.). $a, b$, c indicate a significant decrease $(a, P<0.05 ; b, P<0.01$; $c, P<0.001)$ in ${ }^{3} \mathrm{H}$-thymidine incorporation in enoxaparin-treated cells compared to untreated cells.

A.

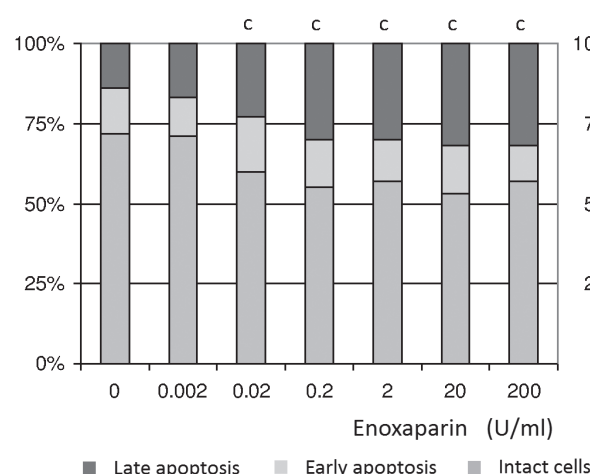

B.

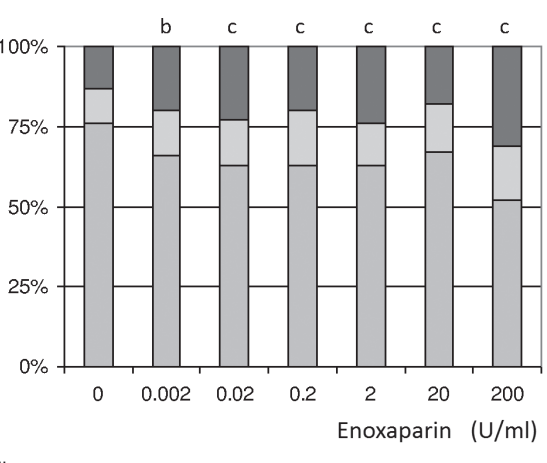

Figure 2. Quantification of enoxaparin-induced cell death

To simplify result presentation, the percentage of unlabeled cells (intact cells) and those labeled with Annexin $\mathrm{V}$ (early apoptosis) and labeled with Annexin V and/or propidium iodate (late apoptosis and necrosis) were shown on the same figures. Adult fibroblasts - Panel A Fetal fibroblasts - Panel B. Results represent means \pm S.D. of 3 separate experiments. b, c indicate a significant increase $(P<0.01$; $C$, $P<0.001)$ in the number of cells stained with annexin $\mathrm{V}$ and/or propidium iodide in enoxaparin-treated cells compared to untreated cells. 


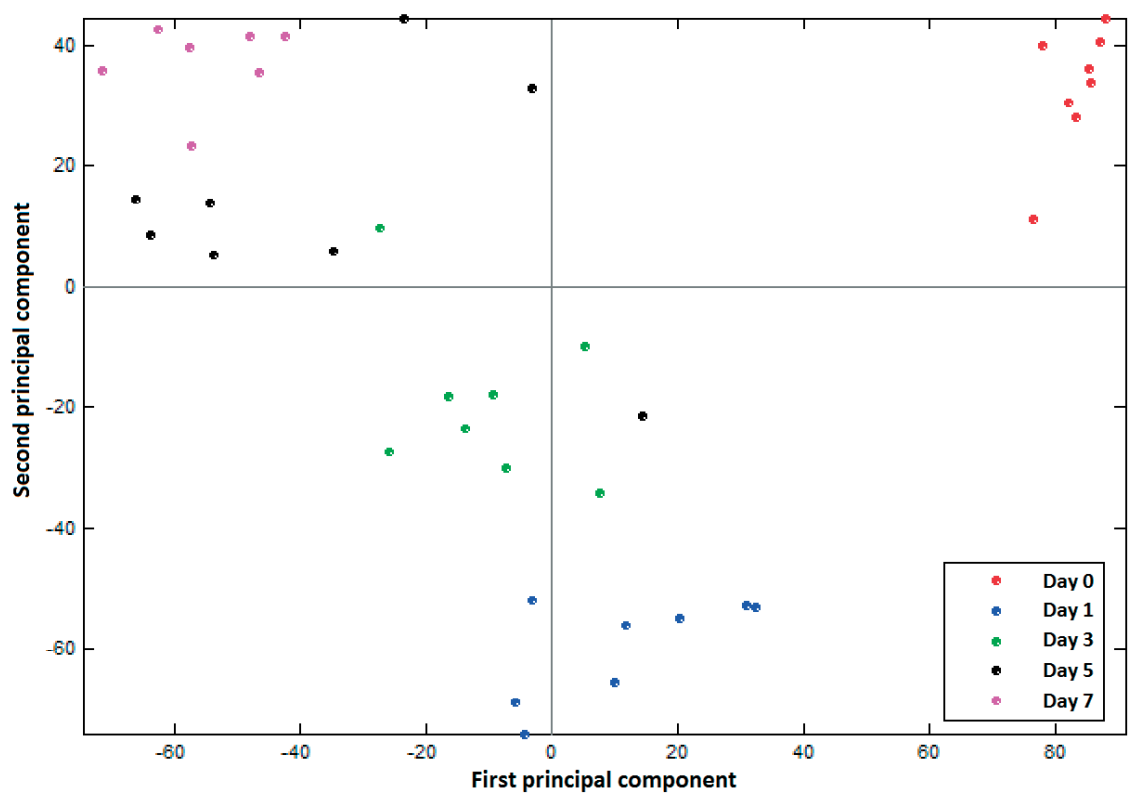

Figure 3. Principal component analysis

The first two principal components were computed from 5476 probe sets differentially changed at least 2-fold at least at one of the analyzed time points.

necrosis with areas of necrosis penetrating to the muscular layer, massive infiltration by PMNs and lymphocytes, and moderate infiltration by macrophages.

On day 7 , there was distinct formation of granulation tissue and numerous blood vessels within the submucosa. In addition, we observed a moderate to marked chronic
A.

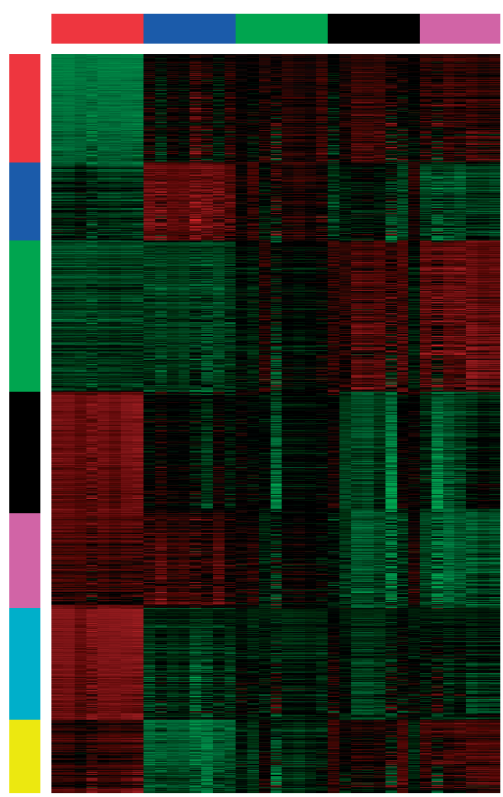

B.
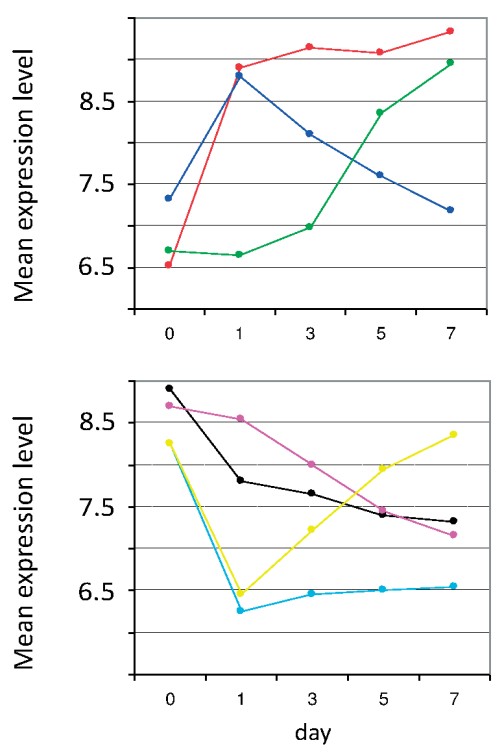

Figure 4. Panel A. Supervised hierarchical clustering from 5476 probe sets differentially changed at least 2-fold at least at one of the analyzed time points yielding 7 distinct clusters of separated expression patterns during the early phase of anastomosis healing (panels A and B)

Across the top, individual tissue samples from day 0 (red), 1 (blue), 3 (green), 5 (black), and 7 (violet) after surgery are arrayed in a column on the left side in rows are shown the top individual probe sets that form the 7 clusters. The color in each cell reflects the expression level of the corresponding probe set in the corresponding array sample relative to its mean level of expression estimated for the entire set of samples. Red indicates expression levels greater than means, and green indicates lower than means.

Panel B. Temporal expression patterns of genes identified by hierarchical clustering, as shown in Panel A inflammatory response and restitution of mucosal epithelial layers, while edema had almost disappeared.

In the specimens obtained from post-operative day 5 , the analyzed microscopic features of repair processes were diverse. In two animals, scoring of the anastomosis healing showed submucosalmuscular restoration with features typical for day 7 , while two other rats exhibited a significant delay in anastomotic repair, with all histological features typical for day 3 .

The intensity of histological features of healing did not differ between enoxaparin-treated and control rats at any time point ( $\mathrm{Ta}-$ ble 1).

\section{Gene expression patterns of colonic anastomotic site} analyses based on its poor quality, according to the parameters established by Affymetrix, and quantitative and parallel measurements of gene expression were performed on the remaining 39 arrays. From over 31000 probe sets of the GeneChip Rat Genome 2302.0 microarray, the total number of probe sets after the filtering step was 15749. To test the consistency of the data sets, PCA was used to transform gene expression variables (based on all probe sets with detectable expression levels) from each array to corresponding principal-component scores. The resulting plot (Supplementary Fig. 1) showed that while most arrays from the same experimental day clustered together, four arrays from anastomotic tissue on day 5 post-surgery were notably distinct from the others. The graphical summary of relationships between studied groups of tissue samples was related to results obtained in the microscopic analysis of anastomoses. Furthermore, as in the case of histological features of healing, the graphical evaluation of gene expression did not reveal significant differences between heparin-treated and untreated rats (not shown). 
Table 2. Terms with the highest overrepresentation selected by Gene Ontology functional analysis among genes differentially changed at least at two of the anastomosis healing time points

\begin{tabular}{|c|c|c|c|c|c|}
\hline \multirow[t]{2}{*}{ Ids } & \multirow[t]{2}{*}{ GO description } & \multicolumn{4}{|c|}{$\begin{array}{l}\text { Pair-wise comparisons between days: } \\
\text { (pa.values) }\end{array}$} \\
\hline & & $0-1$ & $0-3$ & $0-5$ & $0-7$ \\
\hline GO: 0006955 & immune response & 0.00012 & $3.10 \mathrm{E}-08$ & 0.000152 & $7.45 \mathrm{E}-12$ \\
\hline GO: 0007626 & locomotory behavior & 0.00012 & 2.87E-09 & $4.81 \mathrm{E}-05$ & $2.18 \mathrm{E}-11$ \\
\hline GO: 0030593 & neutrophil chemotaxis & 0.000263 & $2.53 \mathrm{E}-05$ & 0.001341 & 7.13E-05 \\
\hline GO: 0007267 & cell-cell signaling & 0.000533 & $1.47 \mathrm{E}-05$ & 0.001341 & 0.000554 \\
\hline GO: 0055065 & metal ion homeostasis & 0.00292 & 0.000255 & & 0.001051 \\
\hline GO: 0006968 & cellular defense response & & 0.002067 & 0.005308 & 4.27E-06 \\
\hline GO: 0006935 & chemotaxis & & 0.000653 & 0.005342 & $4.55 \mathrm{E}-06$ \\
\hline GO: 0009653 & anatomical structure morphogenesis & & 0.009583 & 0.00763 & $7.82 \mathrm{E}-06$ \\
\hline GO: 0065008 & regulation of biological quality & & $1.02 \mathrm{E}-05$ & 0.000301 & 2.77E-05 \\
\hline GO: 0007155 & cell adhesion & & 0.000639 & $1.45 \mathrm{E}-06$ & 0.000886 \\
\hline GO: 0001775 & cell activation & & 0.003249 & & 8.97E-05 \\
\hline GO: 0055082 & cellular chemical homeostasis & & 0.001371 & & 0.000886 \\
\hline GO: 0050801 & ion homeostasis & & 0.000771 & & 0.000886 \\
\hline GO: 0009611 & response to wounding & & 0.000587 & 0.001416 & \\
\hline GO: 0016477 & cell migration & & 0.002837 & 0.001641 & \\
\hline GO: 0007275 & multicellular organismal development & & & $3.04 \mathrm{E}-05$ & 4.84E-09 \\
\hline GO: 0006954 & inflammatory response & & & $4.58 \mathrm{E}-05$ & 7.95E-09 \\
\hline GO: 0048771 & tissue remodeling & & & 0.000491 & 2.91E-06 \\
\hline GO: 0002684 & $\begin{array}{l}\text { positive regulation of immune system } \\
\text { process }\end{array}$ & & & 0.001486 & $3.15 \mathrm{E}-05$ \\
\hline GO: 0006959 & humoral immune response & & & 0.006994 & 0.000312 \\
\hline GO: 0009888 & tissue development & & & 0.000373 & 0.000502 \\
\hline
\end{tabular}

To establish gene expression signatures of the early phase of colonic healing, we then combined four arrays from control and four arrays from enoxaparin-treated rats on each testing day. Of the 15749 probe sets, ANOVA revealed 5476 probe sets that differentially changed at least 2 -fold $(F D R=0.001)$ at least at one of the analyzed time points (Fig. 3). Supplementary Table 1 (Suplementary Material available on www.actabp.pl) gives the complete list of the probe sets that were differentially expressed after surgery.

Notably, unsupervised hierarchical clustering of the 5476 probe sets formed seven distinct clusters of clearly separated expression patterns during the early phase of anastomosis healing (Fig. 4A). As shown in Fig. 4B, $28 \%$ of the differentially expressed probe sets showed a continual decrease in expression from the first day, and $15 \%$ of probe sets exhibited a dramatic decline on the first day after surgery with a continual low level of expression on the remaining days. Two pattern vectors consisted of $15 \%$ and $20 \%$ of probe sets and showed a continual increase in transcript levels starting from the first or third day after surgery, respectively. Two other pattern vectors showed a peak increase or peak decrease on the first day followed by a continual recovery to presurgery basal expression levels. They represented $11 \%$ and $10 \%$ of differentially expressed probe sets, respectively.

Functional features of the 5476 differentially expressed transcripts, as analyzed based on their annotation to
Gene Ontology terms, allowed selection of 79 subcategories (Supplementary Table 2). Terms with the highest level of significance were represented by: multicellular organismal development (GO: 0007275), locomotory behavior (GO: 0007626), immune response (GO: 0006952), cell adhesion (GO: 0007155), inflammatory response (GO: 0006954), cell-cell signaling (GO: 0007267), blood vessel development (GO: 0001568), tissue remodeling (GO: 0048771), and phosphate transport (GO: 0006817). Several other GO terms, like cell migration (GO: 0016477), cellular defense response (GO: 0006968), angiogenesis (GO: 0001525), growth (GO: 0040007), cell proliferation (GO: 0008283), leukocyte activation (GO: 0045321), and blood coagulation (GO: 0007596), which exhibited lower levels of statistical significance, were also related to repair mechanisms. Thus, most of the extracted nodes indicated that many of the differentially expressed genes were involved in wound healing.

When the pair-wise comparisons were used, 2502, 2091, 1895, and 3044 of differentially expressed probe sets between normal colon and anastomosis were selected from days 1, 3, 5 and 7 after surgery, respectively (Supplementary Table 3). Of these, respectively $36 \%$, $43 \%, 48 \%$, and $46 \%$ of probe sets were upregulated. Further functional analysis using GO subcategories revealed that genes subdivided according to function were annotated to many terms closely related to tissue repair (Supplementary Table 4; Table 2). 


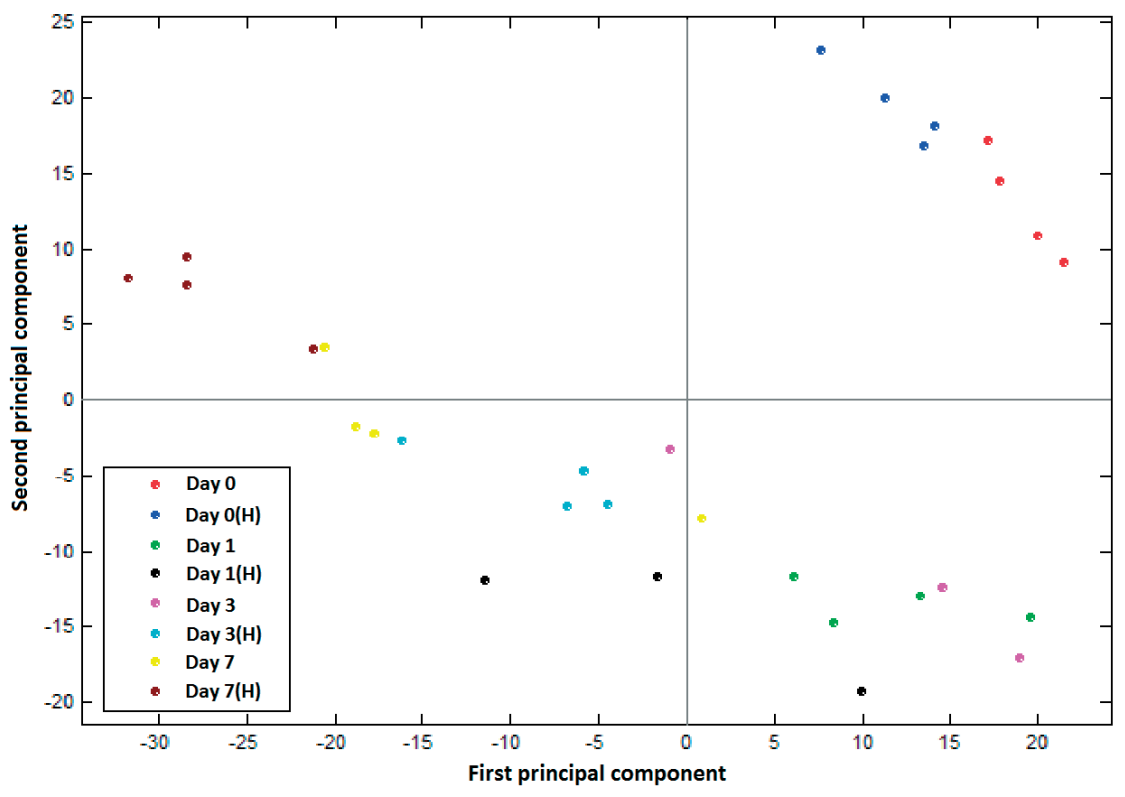

Figure 5. Principal component analysis

The first two principal components were computed from 294 probe sets that were selected from 5475 probe sets distinguishing days of anastomotic site healing $(\mathrm{H})$ - enoxaparin-treated rats.

\section{Enoxaparin influences gene expression profiling of bowel anastomosis sites}

Differences in gene expression between microscopically different transverse cross-sections representing the early anastomosis healing phases were high enough to compensate, at least partly, the biological variability identified by microscopic evaluation. However, when microarray technology was used for screening a potentially few enoxaparin-related genes with relatively small expression level changes among thousands of unaffected genes, it was crucial to extract biological information from properly selected analytical data. Therefore, the choice of microarrays was an important step in further processing of expression data.

Because of an a priori expectation of small differences in expression profiles, the next step of the microarray data analyses was to establish the groups of arrays to be extracted together. Based on imperfect internal consistency across array series (Supplementary Fig. 1), 10 arrays, including those representing day 5 , one array from day 1 , and one array from day 3 after surgery, were rejected from the analysis. They were distinct from arrays that clustered together on their respective day and significantly affected the selection procedure of differentially expressed genes. In addition, we used more permissive criteria $(P$ values $<0.01$ and the fold change set at $>1.25$ or $<0.8$ ).

We estimated 566 out of 15749 probe sets as passing two-way ANOVA statistical testing and exhibiting differentiated anastomosis expression profiles between heparin-treated and control rats, as visualized by PCA (Supplementary Fig. 2; Supplementary Table 5). They included 294 out 5476 probe sets that were selected as showing gene expression patterns of colonic anastomotic sites distinguishing days of healing (Fig. 5; Supplementary Table 6).

Pair-wise comparisons between the heparin-treated and untreated rats revealed 46,229, 116, and 61 probe sets of differentiated gene expression levels $(\mathrm{FC}>1.25)$ on days $0,1,3$, and 7 , respectively. Of these, 83 exhibited at least twofold-change $(>2.0$ or $<0.5) ; 1,45,31$, and 6 probe sets were discriminative between enoxaparin-treated and untreated rats on days $0,1,3$, and 7 , respectively (Supplementary Table 7). Seventy-six probe sets with the highest differences in expression level were upregulated in response to enoxaparin treatment. They represented genes encoding proteins with different functional annotations according to GO, such as inflammatory response [natriuretic peptide precursor type $B$, chemokine (C-C motif) ligand 4, chemokine (C-C motif) receptor 1 , chemokine (C-C motif) receptor-like 2 , chemokine (C-C motif) receptor 5, chemokine (C-C motif) ligand 6]; signal transduction [RAS guanyl releasing protein 4 (similar to Rho-GTPase-activating protein 25), Rho GTPase activating protein 9, phosphodiesterase 4B, caspase recruitment domain family/ member 11, tensin]; regulation of transcription (cAMP responsive element modulator, nuclear factor interleukin 3 regulated, activating transcription factor 3 , early growth response 1 , nuclear receptor subfamily 1 /group $\mathrm{D} / \mathrm{mem}$ ber 1 , nuclear receptor subfamily 1 /group $\mathrm{D} /$ member $2 \mathrm{D}$, site albumin promoter binding protein); regulation of apoptosis (B-cell leukemia/lymphoma 2-related protein A1, complement component 6 , interleukin 8 receptor beta); and angiogenesis (annexin A2, vascular endothelial growth factor A).

Although gene-by-gene statistical testing of microarray data showed that many of the genes affected by enoxaparin appeared to be implicated in anastomosis wound repair, the ontological analysis identified GO subcategories related to heparin treatment only from the list of 120 probe sets. Of these, genes annotated to "cell communication" $(P=4.61 \mathrm{E}-05)$, "signal transduction" $(P=0.000221)$, "cell surface receptor linked signal transduction" $(P=0.002207)$, and "phagocytosis" $(P=0.00612)$ were found to exhibit high levels of statistical significance.

\section{DISCUSSION}

The proper healing of the gut surface requires distinct mechanisms including migration and proliferation of mature epithelial cells and maturation and differentiation of undifferentiated epithelial cells. A deeper wound healing represents the interference and collaboration between epithelial and non-epithelial cell populations in the mucosal and submucosal layers, which are modulated by acute and chronic inflammation (Basson et al., 2003; Sturm et al., 2008). In addition, several coordinated interactions between cells, integrins, extracellular matrix molecules, and metalloproteinases are crucial for the healing process (Steffensen, 2001). All of these processes are primarily related to a spectrum of molecular alterations. 
Until now, evidence regarding wound repair has been mostly obtained from clinical observations supported by histological evaluation. Such studies in combination with cell-oriented molecular studies have been successful in the elucidation of the structure and function of various types of cells involved in hemostasis, removal of cell debris, and prevention of infection, and reflect the immune reaction to injury (Verhofstad et al., 2001). Although molecular aspects of wound healing have been studied both in vitro and in vivo, molecular mechanisms of tissue repair after injury are rather poorly characterized. In vitro studies use a mechanical injury of confluent monolayers of cultured cells (Dayem et al., 2003), while the majority of in vivo studies have been performed with dermal wound models (Cole et al., 2001; Cooper et al., 2005; Colwell et al., 2008).

For the first time, we present a global view of gene expression during the early phase of anastomosis healing. Not surprisingly, thousands of genes display expression changes following anastomotic wounding that relate to key cellular processes, including cell migration, growth, proliferation, and differentiation, which result in rapid wound closure and repair. Because the inflammatory process is a key component of wound healing, numerous genes encoding cytokines and growth factors, prostaglandins, protein kinases, and phosphatases and mediators of DNA synthesis, gene transcription, phospholipid metabolism, or protein synthesis have been found to be differentially expressed during anastomosis healing. Many of these gene expression alterations are similar to those involved in adaptive molecular changes against mucosal injury of the gastrointestinal tract as well as most other injured tissues and organs (Gurtner et al., 2008; Ostrowski et al., 2007; Colwell et al., 2008). Here, microarrays seem to be well suited for molecular fingerprinting of various stages of anastomosis healing.

Though each cell of the body contains the same complete set of genetic information, cells in multicellular organisms are grouped into specific and distinct structures and functions. The cellular diversity results from differential gene expression that adjusts the metabolism of each cell in response to the developmental state, adaptive processes, and environmental factors. Understanding this cellular complexity requires models based on levels of phenotype organization derived from the translation of steady-state analyses into comprehensive models of molecular interactions. Microarray experiments identify and quantify individual elements rather than functional interactions and dynamics among the highly structured systems and generate lists of differentially expressed genes. Thus, the major disadvantage of microarray studies comes from the fact that they do not provide a distinction between gene expression profiling of the various cellular components of the studied tissue. To obtain functional information from microarray data, an ontological analysis approach is usually used to build inter- and intracellular biological networks. Consequently, we analyzed the functional features of differentially expressed genes and the specific pathways involved in anastomosis healing signals using the annotation of functional groups of genes using GO. Although GO has several conceptual limitations (Khatri et al., 2005), it not only allows support from expert information extracted from relevant pathophysiological and pathological studies but also provides novel insights into the molecular aspects of wound healing.

Notably, our analyses may have missed some biological information regarding processes of anastomosis heal- ing, a problem that is attributable to most of the highthroughput analytical platforms. As a consequence of applications for statistically testing a fixed threshold of significance, genes exhibiting smaller expression differences were eliminated from the analysis, although some of them might also be implicated in anastomosis wound repair. In addition, some biologically relevant genes might have been eliminated separately by ontological analysis because this method addresses only those genes that have been previously annotated to the relevant "biological process," "molecular function," and/or "cellular component." Thus, functional analysis of our data remains open ended, and detailed gene expression data including microarray CEL intensity files are publicly available online at http://integromics.pl/files/Enoxaparine/

Our study aimed to identify whether enoxaparin, an LMWH, affects gene expression patterns during the early anastomosis healing. Heparin is an anionic glycosaminoglycan that inhibits anticoagulant activity mediated by antithrombin III, inactivates factors IIa (thrombin), Xa, IXa, XIa, and XIIa, and prevents the transformation of fibrinogen into an insoluble fibrin clot. LMWHs, which are derived from UFH by chemical or enzymatic depolymerization, inactivate factor $\mathrm{Xa}$ and have a minor antifactor IIa effect. Inhibition of coagulation by heparins causes a shift of balance towards fibrinolysis. Plasmin and tissue activator of plasminogen, which act as structural elements of the anticoagulant system, contribute to the activation of collagenase, resulting in increased degradation of extracellular matrix proteins like collagen (Singer et al., 1999). Although heparin treatment did not affect the tensile strength of the anastomosis in rats (Matzsch et al., 1987), prophylactic doses of LMWHs specifically inhibit adhesion processes in the abdomen (Arikan et al., 2005).

The results of this study showed anti-proliferative and late apoptotic/necrotic effects of enoxaparin in cultured fibroblasts, confirming viability alterations found in various other cells in response to UFH and LMWH treatment (Krzesniak-Wszola et al., 2007). In addition, enoxaparin induced significant gene expression changes during the early phases of bowel anastomosis healing in rats, although it did not affect the intensity of histological features of the healing.

Colon anastomosis constitutes a considerable therapeutic challenge. Restoration of tissue integrity and homeostasis depends on a specific genetic program in which closely regulated cross-talk between multiple intercellular and intracellular signaling pathways is activated and synchronized. Whether the LMWH-related cellular and molecular changes may affect the process of anastomosis healing remains to be established. Individual susceptibility to disease is based on genetic variance that determines a patient's defense and adaptive mechanisms to environmental factors, particularly molecular mechanisms. However, intestinal tract continuity is reestablished by a spectrum of repair mechanisms that includes tension-free anastomosis and adequate tissue perfusion in anastomosed stumps, time of operation, intraoperative blood loss, and intraoperative contamination of the operative field, possibly related to the surgeon's experience. The risk of dehiscence also relates to both preoperative and postoperative factors such as age, gender, malnutrition, and cardiovascular diseases (Krzesniak-Wszola et al., 2007). Thus, further studies should be carried out to resolve whether or not heparin treatment may be a risk factor after intestinal surgery, at least in some patients. 


\section{Acknowledgements}

This work was supported by the Polish Ministry of Science and Higher Education grants (PBZMNiI-2/1/2005, 2-P05C-053-30) and the CMKP grant (501-1-1-09-17/05).

\section{REFERENCES}

Affymetrix (2002) GeneChip ${ }^{\circledR}$ Expression Analysis - Data Analysis Fundamentals.

Arikan S, Adas G, Barut G, Toklu AS, Kocakusak A, Uzun H, Kemik O, Daduk Y, Aydin S, Purisa S (2005) An evaluation of low molecular weight heparin and hyperbaric oxygen treatment in the prevention of intra-abdominal adhesions and wound healing. Am J Surg 189: $155-160$.

Ashburner M, Ball CA, Blake JA, Botstein D, Butler H, Cherry JM, Davis AP, Dolinski K, Dwight SS, Eppig JT, Harris MA, Hill DP, Issel-Tarver L, Kasarskis A, Lewis S, Matese JC, Richardson JE, Ringwald M, Rubin GM, Sherlock G (2000) Gene ontology: tool for the unification of biology. The Gene Ontology Consortium. Nat Genet 25: 25-29.

Basson MD (2003) Invited research review: Cell-matrix interactions in the gut epithelium. Surgery 133: 263-267.

Benjamini Y, Hochberg Y (1995) Controlling the false discovery rate: a practical and powerful approach to multiple testing. I Royal Statistical Society 57: 289-300.

Carroll LA, Koch RJ (2003) Heparin stimulates production of bFGF and TGF-beta 1 by human normal, keloid, and fetal dermal fibroblasts. Med Sci Monit 9: BR97-BR108.

Cole J, Tsou R, Wallace K, Gibran N, Isik F (2001) Early gene expression profile of human skin to injury using high-density cDNA microarrays. Wound Repair Regen 9: 360-370.

Collen A, Smorenburg SM, Peters E, Lupu F, Koolwijk P, Van Noorden C, van Hinsbergh VW (2000) Unfractionated and low molecular weight heparin affect fibrin structure and angiogenesis in vitro. Cancer Res 60: 6196-6200.

Colwell AS, Longaker MT, Peter Lorenz H (2008) Identification of differentially regulated genes in fetal wounds during regenerative repair. Wound Repair Regen 16: 450-459.

Cooper L, Johnson C, Burslem F, Martin P (2005) Wound healing and inflammation genes revealed by array analysis of 'macrophageless' PU.1 null mice. Genome Biol 6: R5.

Dayem MA, Moreilhon C, Turchi L, Magnone V, Christen R, Ponzio G, Barbry P (2003) Early gene expression in wounded human keratinocytes revealed by DNA microarray analysis. Comp Funct Genom ics 4: 47-55.

Eberl T, Jagoditsch M, Klingler A, Tschmelitsch J (2008) Risk factors for anastomotic leakage after resection for rectal cancer. Am J Surg 196: $592-598$.

Falcon S, Gentleman R (2007) Using GOstats to test gene lists for GO term association. Bioinformatics 23: 257-258.

Ferrao AV, Mason RM (1993) The effect of heparin on cell proliferation and type-I collagen synthesis by adult human dermal fibroblasts. Biochim Biophys Acta 1180: 225-230.

Gentleman RC, Carey VJ, Bates DM, Bolstad B, Dettling M, Dudoit S, Ellis B, Gautier L, Ge Y, Gentry J, Hornik K, Hothorn T, Huber W, Iacus S, Irizarry R, Leisch F, Li C, Maechler M, Rossini AJ, Sawitzki G, Smith C, Smyth G, Tierney L, Yang JY, Zhang J (2004) Bioconductor: open software development for computational biology and bioinformatics. Genome Biol 5: R80.

Gurtner GC, Werner S, Barrandon Y, Longaker MT (2008) Wound repair and regeneration. Nature 453: 314-321.
Khatri P, Draghici S (2005) Ontological analysis of gene expression data: current tools, limitations, and open problems. Bioinformatics 21: 3587-3595.

Khorana AA, Sahni A, Altland OD, Francis CW (2003) Heparin inhibition of endothelial cell proliferation and organization is dependent on molecular weight. Arterioscler Thromb Vasc Biol 23: 2110-2115.

Kock HJ, Werther S, Uhlenkott H, Taeger G (2002) [Influence of unfractionated and low-molecular-weight heparin on bone healing: an animal model]. Unfallchirurg 105: 791-796.

Krzesniak-Wszola N, Bielecki K, Ostrowski J (2007) Do low-molecular-weight heparins influence the healing process in colon anastomosis? Colorectal Dis 9: 489-493.

Kuzu MA, Kuzu L, Koksov C, Akryl FH, Uzol D, Kale IT, Orhan D, Terzi C (1998) Histological evaluation of colonic anastomotic healing in the rat following preoperative 5-fluorouracil, fractionated irradiation, and combined treatment. Int J Colorectal Dis 13: 235-240.

Lake AC, Castellot JJ Jr (2003) CCN5 modulates the antiproliferative effect of heparin and regulates cell motility in vascular smooth muscle cells. Cell Commun Signal 1: 5.

Lane DA, Lindahl U (1989) Heparin: chemical and biological properties, clinical applications. CRC Press Boca Raton.

Lustosa SA, Matos D, Atallah AN, Castro AA (2002) Stapled versus handsewn methods for colorectal anastomosis surgery: a systematic review of randomized controlled trials. Sao Paulo Med J 120: 132136.

Makela JT, Kiviniemi H, Laitinen S (2003) Risk factors for anastomotic leakage after left-sided colorectal resection with rectal anastomosis. Dis Colon Rectum 46: 653-60.

Matzsch T, Bergqvist D, Blomquist P, Jiborn H (1987) Influence of standard heparin or low molecular weight heparin on healing of abdominal wounds and colonic anastomoses in rats. Acta Chir Scand 153: $593-8$.

Ostrowski J, Mikula M, Karczmarski J, Rubel T, Wyrwicz LS, Bragoszewski P, Gaj P, Dadlez M, Butruk E, Regula J (2007) Molecular defense mechanisms of Barrett's metaplasia estimated by an integrative genomics. J Mol Med 85: 733-743.

Schmidt O, Merkel S, Hohenberger W (2003) Anastomotic leakage after low rectal stapler anastomosis: significance of intraoperative anastomotic testing. Eur J Surg Oncol 2003 29: 239-243.

Singer AJ, Clark RA (1999) Cutaneous wound healing. N Engl J Med 341: $738-746$.

Steffensen B, Hakkinen L, Larjava H (2001) Proteolytic events of wound-healing - coordinated interactions among matrix metalloproteinases (MMPs), integrins, and extracellular matrix molecules. Crit Rev Oral Biol Med 12: 373-398.

Street J, Power C, Wakai A, Wang JH, McGuinness A, Redmond HP (2000a) Low molecular weight heparins induce osteoblast apoptosis: a putative mechanism for iatrogenic osteopenia. J Bone Joint Surgery 84-B: 7.

Street JT, McGrath M, O'Regan K, Wakai A, McGuinness A, Redmond HP (2000b) Thromboprophylaxis using a low molecular weight heparin delays fracture repair. Clin Orthop Relat Res 278-289.

Sturm A, Dignass AU (2008) Epithelial restitution and wound healing in inflammatory bowel disease. World J Gastroenterol 14: 348-353.

Stumpf M, Klinge U, Wilms A, Zabrocki R, Rosch R, Junge K, Krones C, Schumpelick V (2005) Changes of the extracellular matrix as a risk factor for anastomotic leakage after large bowel surgery. Surgery 137: 229-234.

Tiozzo R, Reggiani D, Cingi MR, Bianchini P, Osima B, Calandra S (1991) Effect of heparin derived fractions on the proliferation and protein synthesis of cells in culture. Thromb Res 62: 177-188.

Wu Z, Irizarry RA, Gentleman R, Martinez-Murillo F, Spencer F (2004) A model based background adjustment for oligonucleotide expression arrays. J Am Stat Ass 99: 909-917.

Verhofstad MH, Lange WP, van der Laak JA, Verhofstad AA, Hendriks T (2001) Microscopic analysis of anastomotic healing in the intestine of normal and diabetic rats. Dis Colon Rectum 44: 423-431. 